\title{
Atributos de solo irrigado com água subterrânea cultivado com mangueiras 'Palmer' no Norte Mineiro'
}

\author{
Fernanda Soares Oliveira ${ }^{2}$, Mauro Franco Castro Mota $^{3}$, Moacir Brito Oliveira ${ }^{4}$, Silvânio Rodrigues \\ dos Santos ${ }^{5}$, Marcos Koiti Kondo ${ }^{5}$, Igor Nogueira de Castro ${ }^{5}$
}

Resumo: A irrigação contínua, principalmente no semiárido pode enriquecer o solo com sais prejudiciais à sua fertilidade, inviabilizando o cultivo de fruteiras devido ao desequilíbrio químico e alteração da disponibilidade de nutrientes. Neste sentido, objetivou-se avaliar os atributos químicos e físico-químicos de um Latossolo Vermelho eutrófico quanto à fertilidade e salinidade, sob mangicultura irrigada com água de aquífero confinado no Norte de Minas Gerais. A amostragem de solo foi realizada nas camadas de 0-0,2;0,2-0,4;0,4-0,6 e 0,6-0,8 m de profundidade, em seis estabelecimentos rurais, contendo duas condições em cada um: a) mata nativa e b) centro da área irrigada por microaspersão, onde foram determinados o pH em água, $\mathrm{Ca}, \mathrm{Mg}, \mathrm{K}, \mathrm{Na}, \mathrm{Al}, \mathrm{H}+\mathrm{Al}, \mathrm{Fe}, \mathrm{Mn}$, $\mathrm{Cu}, \mathrm{B}$ e Zn. Também, na água de irrigação de cada estabelecimento, foram avaliados o $\mathrm{pH}$, condutividade elétrica, $\mathrm{Ca}^{2+}, \mathrm{Mg}^{2+}, \mathrm{Na}^{+}, \mathrm{CO}_{3}{ }^{2-}, \mathrm{HCO}_{3}{ }^{-}, \mathrm{Cl}^{-}$e a RAS. A irrigação com água de aquífero carbonático aumenta os teores de cálcio, eleva o $\mathrm{pH}$ e diminui a disponibilidade do manganês, aumenta a relação $\mathrm{Ca} / \mathrm{Mg}$ e $\mathrm{Ca} / \mathrm{K}$ no solo cultivado com mangueira 'Palmer'.

Palavras-chave: Salinidade; Qualidade da água; Alterações no solo; Mangifera indica.

\section{Characterization of soil irrigated with groundwater in 'Palmer' mangrove orchards in the North of Minas Gerais}

\begin{abstract}
Continuous irrigation, mainly in the semi-arid region, can harm soil fertility with salt improvement, that unbalances soil chemicals and nutrient availability, and make impracticable fruit cropping. This work evaluates soil chemical and physical-chemical properties in a Eutrophic Red Latosol (Oxisol) focused on soil fertility and salinity, in a mango crop irrigated with confined aquifer water in Northern Minas Gerais, Brazil. Six farms were analyzed with soil sampling in 0-0.2; 0.2-0.4; 0.4-0.6 e 0.6-0.8 m depth and two conditions each one: a) native forest; b) center point of micro-sprinkler irrigation area. It was measured soil $\mathrm{pH}, \mathrm{Ca}, \mathrm{Mg}, \mathrm{K}, \mathrm{Na}, \mathrm{Al}$, $\mathrm{H}+\mathrm{Al}, \mathrm{Fe}, \mathrm{Mn}, \mathrm{Cu}, \mathrm{B}$, and $\mathrm{Zn}$. Analysis of irrigation water from each farm provided $\mathrm{pH}$, electrical conductivity, $\mathrm{Ca}^{2+}, \mathrm{Mg}^{2+}, \mathrm{Na}^{+}, \mathrm{CO}_{3}{ }^{2-}, \mathrm{HCO}_{3}{ }^{-}, \mathrm{Cl}^{-}$and sodium-adsorption-ratio (SAR). Irrigation with carbonate aquifer source increases $\mathrm{Ca}, \mathrm{pH}, \mathrm{Ca} / \mathrm{Mg}$ and $\mathrm{Ca} / \mathrm{K}$ ratio and decrease manganese availability in mango 'Palmer' field soil.
\end{abstract}

Keywords: Salinity; Water quality; Soil changes; Mangifera indica.

\footnotetext{
${ }^{1}$ Submetido em 24/02/2019 e aprovado em 08/09/2019;

${ }^{2}$ Faculdade Vale do Gorutuba (FAVAG), Nova Porteirinha, Minas Gerais, Brasil. E-mail: fernandaagro.oliver1 @ hotmail.com - ORCID: 00000002-1467-3818;

${ }^{3}$ Instituto de Ciências Agrárias, Universidade Federal de Minas Gerais,. Montes Claros, Minas Gerais, Brasil. E-mail: maurofrancocastro@yahoo.com.br - ORCID: 0000-0001-5184-2476;

${ }^{4}$ Veredas-Mangaclara Serviços Especializados em Fruticultura, Janaúba, Minas Gerais, Brasil. E-mail: moacirconsultoriamanga@yahoo.com.br - ORCID: 0000-0003-4328-9011;
}

${ }^{5}$ Programa de Pós Graduação em Produção Vegetal no Semiárido, Universidade Estadual de Montes Claros, Janaúba, Minas Gerais, Brasil; Email: silvanio.santos@unimontes.br - ORICD: 0000-0003-0245-9184; marcos.kondo@unimontes.br - ORCID: 0000-0001-6875-4907; igornogueira-ig@hotmail.com - ORCID: 0000-0002-4586-0122.

Agropecuária Técnica, Areia-PB, v. 40, n. 3-4, p. 55-63, 2019

https://doi.org/10.25066/agrotec.v40i3-4.44635 


\section{Introdução}

A região semiárida apresenta intensa insolação e evapotranspiração, bem como baixa disponibilidade de água de boa qualidade para irrigação, tornando-se importante e necessária a utilização de água subterrânea como fonte de suprimento hídrico para complemento ou totalidade da irrigação dos cultivos (Carvalho et al., 2017). Mas, geralmente essa água possui maior condutividade elétrica e razão de adsorção de sódio (RAS), assim como maiores teores de $\mathrm{Ca}^{2+}, \mathrm{Mg}^{2+}, \mathrm{Na}^{+}, \mathrm{HCO}_{3}{ }^{-}$e $\mathrm{Cl}^{-}$(Nunes et al., 2005; Nunes et al., 2013).

Nas condições de alta evapotranspiração e baixas precipitações do semiárido, o uso indiscriminado das águas de poços tubulares, sem o adequado manejo da irrigação, têm provocado o enriquecimento dos solos com sais (Medeiros et al., 2017), que compromete suas características físico-químicas, inviabilizando o cultivo e gerando o abandono de áreas agrícolas.

Nessas condições salinizantes, as altas concentrações de $\mathrm{Ca}^{2+}, \mathrm{HCO}_{3}{ }^{-}$e $\mathrm{Na}^{+}$elevam o $\mathrm{pH}$ do solo, alterando o metabolismo e estado nutricional das plantas, e a dinâmica do $\mathrm{N}$ no sistema solo-planta-atmosfera (Brito et al., 2014). $\mathrm{O}$ uso contínuo destas águas subterrâneas pode causar desequilíbrios nutricionais devido às alterações nas relações $\mathrm{Ca}^{2+} / \mathrm{K}^{+}, \mathrm{Ca}^{2+} / \mathrm{Cu}^{2+}$, $\mathrm{Fe}^{2+} / \mathrm{Zn}^{2+}$ e $\mathrm{Zn}^{2+} / \mathrm{H}_{2} \mathrm{PO}_{4}^{-}$, precipitação do fósforo ligado ao cálcio e redução da disponibilidade de micronutrientes como B, $\mathrm{Zn}, \mathrm{Cu}$ e Fe (Malavolta et al., 1997).

No Norte de Minas Gerais, com a implantação dos Perímetros Irrigados do Jaíba e do Gorutuba, houve grande crescimento da agricultura irrigada, inclusive fora das áreas desses perímetros, à partir da utilização de águas subterrâneas, captadas em poços tubulares, nos municípios de Jaíba, Janaúba e Nova Porteirinha (Nunes et al., 2008). Nesta região, a fruticultura é a principal atividade agrícola irrigada, sendo crescente a mangicultura, favorecida pelas condições climáticas e qualidade do solo, quase sempre eutróficos.

A manga é de grande representatividade no mercado interno e uma das principais frutas do comércio externo brasileiro, com receita de US\$ 205 milhões, equivalentes a 179 mil toneladas exportadas em 2017 (Anuário Brasileiro de Fruticultura, 2018). Os principais polos de produção estão nas regiões de Petrolina (PE),
Juazeiro (BA), Livramento de Nossa Senhora, Monte Alto e Taquaritinga (SP), Jaíba e Janaúba (MG). Nas regiões de Jaíba e Janaúba são cultivados 5,1 mil hectares, sendo 610 hectares da cultivar Palmer, irrigados com água subterrânea (Abanorte, 2014) de aquíferos cársticos.

A tolerância da mangueira ao estresse salino varia de média a baixa, sendo a cultivar Palmer medianamente tolerante (Lucena et al., 2012). Apesar dos efeitos adversos no solo, oriundos da irrigação com água subterrânea calcária, há uma escassez de informações que permitam caracterizar corretamente o fenômeno da salinização.

Assim, objetivou-se avaliar os atributos químicos e físico-químicos de um Latossolo Vermelho eutrófico quanto à fertilidade e salinidade, sob mangicultura irrigada com água de aquífero confinado no Norte de Minas Gerais.

\section{Material e Métodos}

O experimento foi realizado nos municípios de Janaúba e Jaíba, região Norte de Minas Gerais, inserida na área do semiárido brasileiro, entre os paralelos $15^{\circ} 18^{\prime}$ a $15^{\circ} 54^{\prime} \mathrm{S}$ e $43^{\circ} 06^{\prime}$ a $43^{\circ} 30^{\prime} \mathrm{W}$, $516 \mathrm{~m}$ de altitude. O clima da região, na classificação de Köppen, é do tipo "Aw" (tropical, com inverno seco). Os valores médios anuais dos elementos do clima são: $873,5 \mathrm{~mm}$ de precipitação anual média, $24,7^{\circ} \mathrm{C}$ de temperatura média e $65 \%$ de umidade relativa do ar.

A geologia regional compreende rochas carbonáticas de origem marinha, do Grupo Bambuí, Subgrupo Paraopeba, referente ao final do Cryogeniano (Neoproterozóico), com 650 milhões de anos, que são intensamente perfuradas para exploração das águas subterrâneas. Estas rochas carbonáticas eventualmente são sobrepostas por coberturas detríticas, em parte colúvio-eluviais e lateríticas do final do Neogeno (Cenozóico), com aproximadamente 1,75 milhão de anos, que formam solos de textura média a argilosa. Ao longo das margens dos rios, predominam os depósitos aluviais mais recentes, do período Quaternário (Iglesias e Uhlein, 2009).

Para avaliar o efeito da irrigação sobre os atributos químicos e físico-químicos do solo foram selecionados seis estabelecimentos rurais com cultivo de mangueira 'Palmer', sendo três áreas comerciais no município de Janaúba e três 
áreas comerciais no município de Jaíba, todas usuárias de água de poços tubulares.

As adubações da mangueira 'Palmer' seguiram a necessidade da cultura, de acordo com a análise de solo e folha, realizada a cada semestre, seguindo a recomendação de Pinto et al. (2002). As fontes de nutrientes foram: sulfato de magnésio, sulfato de amônio e fosfato monoamônio (MAP) como fonte de magnésio, nitrogênio e fósforo, respectivamente. As adubações foram divididas em 03 etapas: $1^{\underline{a}}$ ) reposição ou base, em dose única, logo após a colheita; $2^{a}$ ) desenvolvimento vegetativo, duas aplicações durante o período de renovação da massa foliar; $3^{\mathrm{a}}$ ) pegamento, desenvolvimento e enchimento de frutos, realizado em cinco aplicações. As últimas aplicações ocorreram no final do ciclo, aproximadamente 30 dias antes da coleta de amostras de solo.

A irrigação foi feita com turno de rega de dois dias, determinando-se a demanda hídrica da cultura (ETc), utilizando-se o modelo de
Hargreaves-Samani (Allen et al., 2006) para o cálculo da ETo e coeficiente de cultura $(\mathrm{Kc})$ variando entre 0,57 e 0,89 , de acordo com a fase de cultivo (Santos et al., 2016). Estimou-se a eficiência de aplicação (Ea) mediante avaliações de uniformidade de irrigação a campo (Bernardo et al., 2006). Com os resultados de ETc, Ea e vazão média de emissores, foram calculadas as lâminas líquidas, lâminas brutas acumuladas no turno de rega e tempos de funcionamento do sistema de irrigação. Decorridas as aplicações, as lâminas líquidas de AR foram recalculadas a partir dos tempos de funcionamento, totalizando a lâmina média de $1.200 \mathrm{~mm}$ anuais, sem a contabilização das precipitações efetivas. Resultados da análise da água subterrânea utilizada estão apresentados na tabela 1 , com valores médios do $\mathrm{pH}$ da água, condutividade elétrica $\left(\mathrm{CE}_{\mathrm{a}}\right)$, razão de adsorção de sódio (RAS), relação $\mathrm{Ca} / \mathrm{Mg}$, teores de $\mathrm{K}, \mathrm{Ca}, \mathrm{Na}$ e a classificação quanto ao risco de salinidade (CSA) e sodicidade (CSO).

Tabela 1 Valores médios do $\mathrm{pH}$, condutividade elétrica $\left(\mathrm{CE}_{\mathrm{a}}\right)$, razão de adsorção de sódio (RAS), relação Ca/Mg, $\mathrm{K}^{+}, \mathrm{Ca}^{2+}, \mathrm{Mg}^{2+}, \mathrm{Na}^{+}$, classe quanto ao risco de salinidade (CSA) e sodicidade (CSO) da água subterrânea

\begin{tabular}{|c|c|c|c|c|c|c|c|c|c|}
\hline $\mathrm{pH}$ & $\begin{array}{c}\mathrm{CE}_{\mathrm{a}} \\
\mathrm{dS} \mathrm{m}^{-1}\end{array}$ & $\begin{array}{c}\text { RAS } \\
\mathrm{mmol} \mathrm{L}^{-1}\end{array}$ & $\mathrm{Ca} / \mathrm{Mg}$ & $\mathrm{K}^{+}$ & $\begin{array}{c}\mathrm{Ca}^{2+} \\
\mathrm{mol}_{\mathrm{c}} \mathrm{L}\end{array}$ & $\mathrm{Mg}^{2+}$ & $\mathrm{Na}^{+}$ & CSA & $\mathrm{CSO}$ \\
\hline 7,1 & 1,08 & 0,201 & 4,2 & 0,001 & 0,53 & 0,15 & 0,08 & $\mathrm{C}_{2}$ & $\mathrm{~S}_{1}$ \\
\hline
\end{tabular}

$\mathrm{C}_{2}$ e $\mathrm{S}_{1}=$ respectivamente, risco médio e baixo da água salinizar e sodificar o solo (Ayers \& Westcot, 1999).

Em setembro de 2014, em cada estabelecimento rural, foram coletadas dez amostras simples de solo nas camadas de 0-0,2; 0,2-0,4; 0,4-0,6 e 0,6-0,8 $\mathrm{m}$ de profundidade, em duas áreas: a) condição de sequeiro, com mata nativa $(\mathrm{MN}) \mathrm{e}, \mathrm{b})$ centro da área irrigada por microaspersão (IRR), com pontos de coleta sob a projeção da copa da mangueira com idade entre 8 e 9 anos. Cerca de $1 \mathrm{~kg}$ das amostras compostas de cada camada e cada local amostrado foram enviadas para o laboratório de solos, sendo submetidas às análises de $\mathrm{pH}$ em água, teor de $\mathrm{MO}, \mathrm{Ca}, \mathrm{Mg}, \mathrm{K}, \mathrm{Na}, \mathrm{H}+\mathrm{Al}, \mathrm{Fe}, \mathrm{Mn}, \mathrm{Cu}, \mathrm{B}$ e Zn, segundo o método da Embrapa (2017). Ainda se avaliou a capacidade de troca de cátions potencial (CTC), saturação por bases (V), condutividade elétrica do estrato saturado do solo ( $\mathrm{CE}_{\mathrm{es}}$ ), porcentagem de sódio trocável (PST) e a relação $\mathrm{Ca} / \mathrm{K}$.

$\mathrm{Na}$ ocasião da amostragem de solo, também foram coletadas amostras de água nas saídas dos emissores dos sistemas de irrigação, obedecido o tempo de funcionamento inicial de 40 minutos, sendo armazenadas em recipientes plásticos e, posteriormente, determinando-se o $\mathrm{pH}$; condutividade elétrica da água $\left(\mathrm{CE}_{\mathrm{a}}\right)$; cátions $\left(\mathrm{Ca}^{2+}, \mathrm{Mg}^{2+}, \mathrm{K}^{+} \mathrm{Na}^{+}\right)$e ânions $\left(\mathrm{Cl}^{-}, \mathrm{CO}_{3}{ }^{2+}{\mathrm{e} \mathrm{HCO}_{3}}^{-}\right.$ ), segundo Apha et al. (2012).

Os ânions foram utilizados somente para auxiliar na classificação da água quanto aos riscos de salinidade e sodicidade do solo, proposto por (Ayers \& Westcot, 1999), e os cátions utilizados para calcular a razão de adsorção de sódio (RAS) e a relação $\mathrm{Ca} / \mathrm{Mg}$, conforme descrito por Faria et al. (2009).

As comparações do efeito da fonte de água utilizada sobre os atributos químicos e físicoquímicos do solo foram feitas pelo teste de hipótese $\mathrm{t}$ de Student a 5\% de significância, para dados pareados (solos sob mata nativa - MN e mangueiras irrigadas com água subterrânea - 
IRR) em cada camada amostrada, considerando cada estabelecimento rural como uma repetição.

\section{Resultados e Discussão}

As águas subterrâneas apresentam risco médio de salinidade (Tabela 1), devido às altas concentrações de sais contidos na água, principalmente bicarbonatos $\left(6,48 \mathrm{mmol}_{\mathrm{c}} \mathrm{dm}^{-3}\right.$, valor médio observado). Porém, a salinidade média nos solos irrigados depende da qualidade da água, fatores edafoclimáticos e do manejo da irrigação e salinidade média ao longo do tempo a melhor característica a que a cultura responde. (Porto Filho et al., 2011).

Em relação aos problemas de infiltração por sodicidade são consideradas de baixo risco (S1). Considerando a concentração média de sódio $\left(0,08 \mathrm{mmol}_{\mathrm{c}} \mathrm{dm}^{-3}\right.$, Tabela 1) e uma lâmina média anual de $1200 \mathrm{~mm}$ para cultura da manga, é aportado ao solo $22 \mathrm{~kg} \mathrm{ha}^{-1} \mathrm{ano}^{-1}$ de sódio, podendo elevar em $11 \mathrm{mg} \mathrm{dm}^{-3}\left(0,05 \mathrm{cmol}_{\mathrm{c}} \mathrm{dm}^{-3}\right)$ a cada ano na camada de $0-0,2 \mathrm{~m}$. No entanto, ao avaliar o solo da mata nativa (Tabela 2 ) percebese que não diferiu do solo irrigado. Possivelmente essa quantidade acrescida ao solo tenha sido lixiviada no período chuvoso.
Verificou-se valor médio da relação $\mathrm{Ca}^{2+} / \mathrm{Mg}^{2+}$ igual a 4,2 (Tabela 1). A magnitude deste valor indica a disponibilidade do cálcio devido à sua predominância como cátion e evidencia que com o valor de $\mathrm{pH}$ médio igual a 7,1 , pode ocorrer precipitação de cálcio como carbonato de cálcio. Além disso, o uso dessa água em irrigação pode levar o solo ao desequilíbrio devido à predominância de cálcio. Embora, Raij et al. (1996) relatam que a relação $\mathrm{Ca} / \mathrm{Mg}$ não é um fator limitante para o crescimento e produtividade das culturas, desde que os teores de Ca e Mg estejam acima dos níveis críticos para as plantas, podendo variar de 0,5/1 a 30/1.

$\mathrm{O}$ aumento do $\mathrm{pH}$ na camada superficial da mata nativa, igualando-se à área irrigada (Tabela 2), está associado à ciclagem de nutrientes de camadas mais profundas para a superfície, acumulando as bases. A deposição de resíduos vegetais tende a promover a diminuição da acidez do solo na camada superficial (Maluf et al., 2015), por meio da troca ou complexação dos íons $\mathrm{H}^{+} \mathrm{e}$ $\mathrm{Al}^{3+}$, por $\mathrm{Ca}, \mathrm{Mg}, \mathrm{K}$ e outros compostos presentes no resíduo vegetal, aumentando assim a saturação por bases (Pavinato \& Rosolem, 2008).

Tabela 2 Valores médios de $\mathrm{pH}$ em $\mathrm{H}_{2} \mathrm{O}, \mathrm{Ca}^{2+}, \mathrm{Mg}^{2+}, \mathrm{K}^{+}$e $\mathrm{Na}^{+}$em solos sob mata nativa (MN) e mangueiras irrigadas com água subterrânea (IRR) em Jaíba e Janaúba - MG em diferentes profundidades (Prof)

\begin{tabular}{|c|c|c|c|c|c|c|c|c|c|c|c|c|}
\hline \multirow{2}{*}{ Prof } & \multicolumn{2}{|c|}{$\mathrm{pH} \mathrm{H} \mathrm{H}_{2} \mathrm{O}$} & \multicolumn{2}{|c|}{$\mathrm{H}+\mathrm{Al}$} & \multicolumn{2}{|c|}{$\mathrm{Ca}^{2+}$} & \multicolumn{2}{|c|}{$\mathrm{Mg}^{2+}$} & \multicolumn{2}{|c|}{$\mathrm{K}^{+}$} & \multicolumn{2}{|c|}{$\mathrm{Na}^{+}$} \\
\hline & IRR & MN & $\overline{\mathrm{IRR}}$ & MN & $\overline{\mathrm{IRR}}$ & MN & $\overline{\mathrm{IRR}}$ & MN & $\overline{\mathrm{IRR}}$ & MN & $\overline{\text { IRR }}$ & MN \\
\hline $\mathrm{m}$ & & & -- & & & & $--\mathrm{cmc}$ & $\mathrm{dm}^{-3}$ & & & & \\
\hline $0-0,2$ & $7,3^{\mathrm{a}}$ & $6,8^{\mathrm{a}}$ & $0,83^{\mathrm{a}}$ & $1,63^{\mathrm{a}}$ & $9,50^{\mathrm{a}}$ & $6,53^{\mathrm{b}}$ & $1,46^{\mathrm{a}}$ & $1,58^{\mathrm{a}}$ & $0,40^{\mathrm{a}}$ & $0,51^{\mathrm{a}}$ & $0,15^{\mathrm{a}}$ & $0,10^{\mathrm{a}}$ \\
\hline $0,2-0,4$ & $7,5^{\mathrm{a}}$ & $6,7^{\mathrm{b}}$ & $0,90^{\mathrm{a}}$ & $1,78^{\mathrm{a}}$ & $7,01^{\mathrm{a}}$ & $5,33^{\mathrm{b}}$ & $1,30^{\mathrm{a}}$ & $1,20^{\mathrm{a}}$ & $0,28^{\mathrm{a}}$ & $0,21^{\mathrm{a}}$ & $0,13^{\mathrm{a}}$ & $0,10^{\mathrm{a}}$ \\
\hline $0,4-0,6$ & $7,4^{\mathrm{a}}$ & $6,5^{\mathrm{b}}$ & $1,15^{\mathrm{a}}$ & $1,77^{\mathrm{a}}$ & $6,23^{\mathrm{a}}$ & $5,15^{\mathrm{a}}$ & $1,23^{\mathrm{a}}$ & $1,10^{b}$ & $0,23^{\mathrm{a}}$ & $0,12^{\mathrm{b}}$ & $0,15^{\mathrm{a}}$ & $0,11^{\mathrm{a}}$ \\
\hline $0,6-0,8$ & $7,2^{\mathrm{a}}$ & $5,8^{\mathrm{b}}$ & $1,03^{\mathrm{a}}$ & $2,48^{\mathrm{a}}$ & $6,18^{\mathrm{a}}$ & $4,01^{b}$ & $1,18^{\mathrm{a}}$ & $1,05^{\mathrm{b}}$ & $0,18^{\mathrm{a}}$ & $0,07^{\mathrm{b}}$ & $0,11^{\mathrm{a}}$ & $0,11^{\mathrm{a}}$ \\
\hline $\mathrm{CV}(\%)$ & 11,8 & 13,5 & 15,0 & 48,3 & 18,8 & 21,4 & 21,5 & 23,6 & 46,2 & 62,7 & 25 & 34,3 \\
\hline
\end{tabular}

Médias $(n=6)$ do mesmo atributo, seguidas da mesma letra na linha não diferem entre si pelo teste t a 5\%, com dados pareados.

Para as camadas subsequentes houve diferenças no $\mathrm{pH}$ do solo, sendo observado uma elevação do $\mathrm{pH}$ nos solos irrigados, cujo $\mathrm{pH}$ médio foi de 6,33 e 7,37 para área de mata nativa e irrigada, respectivamente (Tabela 2), o que pode ser atribuído às altas concentrações de carbonato de cálcio, corroborando com os resultados de Nunes et al. (2008). Esses autores observaram valores semelhantes de $\mathrm{pH}$ do solo, quando da aplicação de água subterrânea em área de bananicultura na mesma região, podendo o efeito ser agravado quando não se faz o manejo da irrigação e o controle de salinização do solo.

Apesar da alteração da aparente redução da acidez potencial $(\mathrm{H}+\mathrm{Al})$ (Tabela 2), o teste estatístico aplicado não se mostrou sensível o suficiente para comprovar tal diferença.

$\mathrm{O} \mathrm{Ca}^{2+}$ aportado ao solo via água de irrigação (Tabela 1), elevou o conteúdo deste elemento em $38 \%$ na camada de $0-0,8 \mathrm{~m}$, em relação à mata nativa (Tabela 2). Apesar de não ter sido comparado entre camadas, nota-se tendência de 
diminuição do $\mathrm{Ca}^{+2}$ em profundidade, devido a sua baixa mobilidade no solo. Comportamento semelhante também foi constatado por Nunes et al. (2005) em solo de bananais irrigados com águas subterrâneas na região de Janaúba.

Não houve diferença significativa nos teores $\mathrm{Mg}^{2+}$ e $\mathrm{K}^{+}$na camada de 0 a $0,40 \mathrm{~m}$ (Tabela 2), mesmo com os altos teores destes elementos na água, provavelmente a absorção pela cultura e as perdas por lixiviação seriam equivalentes ao aporte via água de irrigação. Abaixo de $0,4 \mathrm{~m}$, o $\mathrm{Mg}^{2+}$ aumentou aproximadamente $12 \%$ nas duas camadas e o $\mathrm{K}^{+}$aumentou $92 \%$ e $157 \%$ nas camadas de 0,4-0,6 m e 0,6-0,8 m, respectivamente, na área irrigada quando comparados com a mata nativa (Tabela 2).

Variações em profundidade resultam da maior concentração, até $0,4 \mathrm{~m}$ de profundidade, de raízes de absorção da mangueira, que intensificam a exportação dos nutrientes (Almeida et al., 2009). Ainda para o $\mathrm{K}^{+}$, a contribuição mínima da água subterrânea no seu aporte, associado ao deslocamento do complexo de troca provocado pelas altas concentrações de $\mathrm{Ca}^{2+}$ e $\mathrm{Mg}^{2+}$ e consequente elevação da relação $\mathrm{Ca} / \mathrm{Mg}$, reduziram sua disponibilidade nas camadas superficiais, tornando-se semelhante aos teores da mata nativa (Santos et al., 2005).
Não se observou aumento dos teores de $\mathrm{Na}^{+}$no solo irrigado em nenhuma das camadas analisadas (Tabela 2). Possivelmente, isso aconteceu devido à lixiviação com as lâminas de irrigação utilizadas. Em áreas cultivadas, as plantas estimulam a atividade biológica na zona radicular, elevando a concentração de $\mathrm{CO}_{2} \mathrm{e}$ a taxa de dissolução de carbonatos a um nível suficiente para a remoção de parte do $\mathrm{Na}^{+}$ trocável, deixando-o livre na solução para que seja lixiviado, corrigindo o solo (Carmona et al., 2010).

$\mathrm{O} \mathrm{Na}+$ não acumulou com o uso da água subterrânea porque, dentre os cátions trocáveis, o $\mathrm{Na}^{+}$é o último elemento em seletividade de adsorção, devido a sua monovalência e maior raio iônico hidratado (série liotrópica). Assim, quando na mesma concentração dos cátions comuns, ele será o último a ser adsorvido às cargas elétricas, após o preenchimento por $\mathrm{H}^{+}>>>\mathrm{Al}^{3+}>\mathrm{Ca}^{2+}>$ $\mathrm{Mg}^{2+}>\mathrm{K}^{+}>\mathrm{NH}_{4}{ }^{+}>\mathrm{Na}^{+}$(Holanda et al., 2010).

Genericamente, a área irrigada apresentou maiores valores de CTC, V, CE $\mathrm{es}_{\mathrm{es}}$ RAS e PST (Tabela 3). Embora estes dois últimos não diferiram da área com vegetação nativa em nenhuma das profundidades, possivelmente em virtude da baixa concentração de sódio no complexo de troca do solo (Tabela 2).

Tabela 3 Valores médios de capacidade de troca de cátions (CTC), saturação por bases (V), condutividade elétrica do extrato saturado do solo ( $\mathrm{CE}_{\mathrm{es}}$ ), razão de adsorção de sódio (RAS) e porcentagem de sódio trocável (PST) em solos sob mata nativa (MN) e irrigados com água de poços tubulares na mangicultura (IRR) em Jaíba e Janaúba MG em diferentes profundidades (Prof)

\begin{tabular}{|c|c|c|c|c|c|c|c|c|c|c|}
\hline \multirow{2}{*}{ Prof } & \multicolumn{2}{|c|}{ CTC } & \multicolumn{2}{|c|}{ V } & \multicolumn{2}{|c|}{$\mathrm{CE}_{\mathrm{es}}$} & \multicolumn{2}{|c|}{ RAS } & \multicolumn{2}{|c|}{ PST } \\
\hline & IRR & MN & IRR & $\mathrm{MN}$ & IRR & $\mathrm{MN}$ & IRR & $\mathrm{MN}$ & IRR & $\mathrm{MN}$ \\
\hline $\mathrm{m}$ & \multicolumn{2}{|c|}{$--\mathrm{cmol}_{\mathrm{c}} \mathrm{dm}^{3}$} & \multicolumn{2}{|c|}{------\% ------ } & \multicolumn{2}{|c|}{--- dS m ${ }^{-1}---$} & \multicolumn{2}{|c|}{$\left(\mathrm{cmol}_{\mathrm{c}} \mathrm{dm}^{-3}\right)^{0,5}$} & \multicolumn{2}{|c|}{----- \% ---- } \\
\hline $0-0,2$ & $12,4^{\mathrm{a}}$ & $10,2^{b}$ & $92^{\mathrm{a}}$ & $85^{\mathrm{a}}$ & $1,31^{\mathrm{a}}$ & $0,83^{\mathrm{a}}$ & $0,06^{\mathrm{a}}$ & $0,05^{\mathrm{a}}$ & $1,20^{\mathrm{a}}$ & $1,01^{\mathrm{a}}$ \\
\hline $0,2-0,4$ & $8,8^{\mathrm{a}}$ & $9,5^{\mathrm{b}}$ & $99^{\mathrm{a}}$ & $72^{b}$ & $1,03^{\mathrm{a}}$ & $0,53^{\mathrm{b}}$ & $0,06^{\mathrm{a}}$ & $0,05^{\mathrm{a}}$ & $1,38^{\mathrm{a}}$ & $1,21^{\mathrm{a}}$ \\
\hline $0,4-0,6$ & $9,0^{\mathrm{a}}$ & $8,2^{\mathrm{b}}$ & $87^{\mathrm{a}}$ & $79^{\mathrm{a}}$ & $0,88^{\mathrm{a}}$ & $0,51^{\mathrm{a}}$ & $0,07^{\mathrm{a}}$ & $0,06^{\mathrm{a}}$ & $1,71^{\mathrm{a}}$ & $1,43^{\mathrm{a}}$ \\
\hline $0,6-0,8$ & $8,7^{\mathrm{a}}$ & $7,7^{\mathrm{b}}$ & $87^{\mathrm{a}}$ & $68^{\mathrm{b}}$ & $0,95^{\mathrm{a}}$ & $0,61^{\mathrm{a}}$ & $0,06^{\mathrm{a}}$ & $0,07^{\mathrm{a}}$ & $1,38^{\mathrm{a}}$ & $1,57^{\mathrm{a}}$ \\
\hline $\mathrm{CV}(\%)$ & 9,77 & 11,9 & 20,08 & 16,90 & 50,44 & 83,64 & 36,25 & 32,90 & 30,88 & 34,91 \\
\hline
\end{tabular}

Médias ( $\mathrm{n}=6)$ do mesmo atributo, seguidas da mesma letra na linha não diferem entre si pelo teste $\mathrm{t}$ a $5 \%$, com dados pareados.

O solo sob irrigação apresentou maior CTC em todas as profundidades (Tabela 3), em decorrência do incremento de $\mathrm{Ca}^{2+}$ pela água subterrânea. $\mathrm{O} \mathrm{Ca}^{2+}$ participa da CTC em $76 \%$, $81 \%, 69 \%$ e $71 \%$ nas camadas $0-0,2 ; 0,2-0,4 ; 0,4-$ 0,6 e $0,6-0,8 \mathrm{~m}$, respectivamente, além disso, o $\mathrm{pH}$ elevado também contribui para o aumento da
CTC, e isto porque, quando ocorre a diminuição da acidez, a quantidade de íons de hidrogênio em solução também diminui, fazendo com que grupos hidoxílicos se dissociem. Assim, com o decréscimo da acidez, íons de hidrogênio são neutralizados ou removidos dos bordos das partículas coloidais para compensar a diminuição 
desses íons na solução do solo, resultando em novas cargas negativas (Lepsch, 2011).

Quanto à saturação por bases (V), o comportamento foi semelhante ao observado para os teores de $\mathrm{Ca}$ e $\mathrm{Mg}$ trocáveis, aumentando em decorrência da presença dessas bases, adicionadas pela água de irrigação. A exceção da camada de 0,2-0,4 m, não se verificou aumento nos valores de $\mathrm{CE}_{\text {es }}$ (Tabela 3 ), possivelmente em função dos baixos teores de sais solúveis, que tendem a permanecer na forma de precipitados. A diferença observada na camada de $0,2-0,4$ m pode ser atribuída ao acúmulo de sais nas duas primeiras camadas do solo irrigado, já no solo da mata nativa os íons tendem a se concentrar apenas na primeira.

A disponibilidade de micronutrientes é muito variável em função do manejo e do equilíbrio químico do solo. Além disso, é muito sensível aos métodos analíticos o que explica os altos coeficientes de variação (Tabela 4 e 5 ), e consequentemente menor sensibilidade em encontrar diferenças entre as áreas irrigada e de mata nativa. Outro fator que pode ter contribuído foi o uso de extrator ácido (Mehlich-1) que extrai formas complexadas, indisponíveis para as plantas.

Tabela 4 Valores médios, por camada estudada, de $\mathrm{Zn}, \mathrm{Cu}, \mathrm{Fe}, \mathrm{Mn}$ e B extraíveis, em ambiente de mata nativa (MN) e irrigados (IRR), dos solos cultivados com manga em função da água de irrigação em diferentes profundidades (Prof)

\begin{tabular}{|c|c|c|c|c|c|c|c|c|c|c|}
\hline \multirow{2}{*}{ Prof } & \multicolumn{2}{|c|}{$\mathrm{Zn}$} & \multicolumn{2}{|c|}{$\mathrm{Cu}$} & \multicolumn{2}{|c|}{$\mathrm{Fe}$} & \multicolumn{2}{|c|}{$\mathrm{Mn}$} & \multicolumn{2}{|c|}{ B } \\
\hline & IRR & MN & IRR & MN & IRR & MN & IRR & $\bar{M} N$ & IRR & MN \\
\hline $\mathrm{m}$ & & & & & $m$ & $\mathrm{dm}^{3}---$ & & & & - \\
\hline $0-0,2$ & $5,82^{\mathrm{a}}$ & $3,21^{\mathrm{b}}$ & $2,8^{\mathrm{a}}$ & $1,86^{\mathrm{a}}$ & $13,68^{\mathrm{a}}$ & $15,26^{\mathrm{a}}$ & $14,24^{b}$ & $87,85^{\mathrm{a}}$ & $0,71 \mathrm{a}$ & $0,56^{\mathrm{a}}$ \\
\hline $0,2-0,4$ & $1,41^{\mathrm{a}}$ & $0,75^{\mathrm{b}}$ & $1,7^{\mathrm{a}}$ & $1,46^{\mathrm{b}}$ & $21,56^{\mathrm{a}}$ & $19,13^{\mathrm{a}}$ & $68,86^{\mathrm{a}}$ & $39,91^{\mathrm{a}}$ & $0,51 \mathrm{a}$ & $0,43^{\mathrm{a}}$ \\
\hline $0,4-0,6$ & $0,75^{\mathrm{a}}$ & $0,56^{\mathrm{a}}$ & $1,6^{\mathrm{a}}$ & $1,26^{\mathrm{a}}$ & $23,71^{\mathrm{a}}$ & $24,78^{\mathrm{a}}$ & $34,96^{\mathrm{a}}$ & $22,23^{\mathrm{a}}$ & $0,50 \mathrm{a}$ & $0,28^{\mathrm{a}}$ \\
\hline $0,6-0,8$ & $0,40^{\mathrm{a}}$ & $0,48^{\mathrm{a}}$ & $1,4^{\mathrm{a}}$ & $1,10^{\mathrm{b}}$ & $17,40^{\mathrm{a}}$ & $25,20^{\mathrm{a}}$ & $14,70^{\mathrm{a}}$ & $17,15^{\mathrm{a}}$ & $0,28 \mathrm{a}$ & $0,26^{\mathrm{a}}$ \\
\hline $\mathrm{CV}(\%)$ & 29,52 & 44,70 & 18,6 & 25,88 & 41,73 & 34,21 & 34,08 & 43,90 & 60,03 & 79,12 \\
\hline
\end{tabular}

Médias ( $\mathrm{n}=6$ ) do mesmo atributo, seguidas da mesma letra na linha não diferem entre si pelo teste $\mathrm{t}$ a $5 \%$ com dados pareados.

De maneira geral, não houve diferença entre os manejos ao avaliar os micronutrientes, exceto o $\mathrm{Zn}$ (camadas $0-0,2$ e $0,4 \mathrm{~m}$ ), $\mathrm{Cu}$ (camada $0,2-$ $0,4 \mathrm{~m}$ e $0,6-0,8 \mathrm{~m}$ ) e Mn (camada 0,2-0,4 m) (Tabela 4). Este último diferenciou na primeira camada, sendo maior na área de mata nativa. Essa região em estudo, tem um histórico de teores elevados de $\mathrm{Mn}$, possivelmente devido ao material de origem ter muito $\mathrm{Mn}$ em sua composição (Carbonato, $\mathrm{MnCO}_{3}$; pirolusita $\left(\mathrm{Mn}^{4+}\right)$; maganita $\left(\mathrm{Mn}^{3+}\right)$ (Filho et al., 2011). Além disso, o ataque ácido do extrator Mehlich-1 pode ter superestimado os valores de $\mathrm{Mn}$ disponível.

Também, a redução dos teores de Mn na área irrigada pode ser atribuída ao aumento do $\mathrm{pH}$, em virtude do uso de água alcalina $(\mathrm{pH}>7)$. $\mathrm{O}$ aumento dos teores de carbonato de cálcio e magnésio reduz o fluxo difusivo de $\mathrm{Zn}, \mathrm{Fe}, \mathrm{Cu}$ e Mn (Tabela 4), sendo esse decréscimo mais acentuado nas formas catiônicas, devido ao aumento do $\mathrm{pH}$, provocando hidroxilação e formação de espécies metálicas de baixa solubilidade (Pegoraro et al., 2006).

$\mathrm{O}$ maior teor de $\mathrm{Zn}$ na área irrigada pode ser atribuído ao efeito residual de adubação na cultura da manga. Além disso, a maior atividade microbiológica em função dos resíduos orgânicos próximo à projeção da copa pode favorecer o aumento do teor de $\mathrm{Zn}$ na solução do solo, em virtude do maior efeito de dissolução e de complexação desse nutriente no solo, a adição de compostos orgânicos ao solo cria um ambiente de redução, mesmo que temporário, diminuindo $o$ suprimento de $\mathrm{O}_{2}$, decorrente da intensa atividade microbiana, e produzindo compostos orgânicos que podem dissolver e manter um teor elevado de Zn em solução (Hue et al. 2001).

$\mathrm{O}$ manejo na área irrigada com água subterrânea, de modo geral, apresentou relação $\mathrm{Ca} / \mathrm{Mg}$ e $\mathrm{Ca} / \mathrm{K}$ elevada, diferenciando da área de mata nativa na camada superficial (Tabela 5). 
Atributos de solo irrigado com água subterrânea cultivado com mangueiras 'Palmer'...

Oliveira et al.

Tabela 5 Valores médios das relações $\mathrm{Ca} / \mathrm{Mg}$ e $\mathrm{Ca} / \mathrm{K}$, por camada estudada, em ambiente de mata nativa(MN) e irrigado (IRR), dos solos da mangicultura agrupados em função da água de irrigação

\begin{tabular}{|c|c|c|c|c|}
\hline \multirow{2}{*}{ Prof } & \multicolumn{2}{|c|}{$\mathrm{Ca} / \mathrm{Mg}$} & \multicolumn{2}{|c|}{$\mathrm{Ca} / \mathrm{K}$} \\
\hline & IRR & MN & IRR & MN \\
\hline $\mathrm{cm}$ & \multicolumn{4}{|c|}{ - } \\
\hline $0-20$ & $6,46^{\mathrm{a}}$ & $4,56^{\mathrm{b}}$ & $23,54^{\mathrm{a}}$ & $19,54^{\mathrm{a}}$ \\
\hline $20-40$ & $5,73^{\mathrm{a}}$ & $4,73^{\mathrm{a}}$ & $25,20^{\mathrm{a}}$ & $46,37^{\mathrm{a}}$ \\
\hline $40-60$ & $5,62^{\mathrm{a}}$ & $5,02^{\mathrm{a}}$ & $28,72^{\mathrm{a}}$ & $70,45^{\mathrm{a}}$ \\
\hline $60-80$ & $5,76^{\mathrm{a}}$ & $4,18^{\mathrm{a}}$ & $45,20^{\mathrm{a}}$ & $75,96^{\mathrm{a}}$ \\
\hline $\mathrm{CV}(\%)$ & 28,82 & 36,45 & 107,08 & 63,62 \\
\hline
\end{tabular}

Médias (n=6) do mesmo atributo, seguidas da mesma letra na linha não diferem entre si pelo teste t a $5 \%$ com dados pareados.

Devido ao uso de água com relação $\mathrm{Ca} / \mathrm{Mg}$ de 6,68 (Tabela 1), houve desequilíbrio no solo, sugerindo que os teores de Ca sejam diminuídos ou elevados os teores de Mg. Carvalho (2007), preconiza que a relação $\mathrm{Ca} / \mathrm{Mg}$ para a cultura da manga seja de 2,5:1. visto que, atualmente, a literatura considera que esta relação deve está entre 4:1 e 8:1 e a relação $\mathrm{Ca} / \mathrm{K}$ não teve diferença significativa entre as áreas, em decorrência dos baixos teores de $\mathrm{K}$ no solo em virtude da absorção pela cultura, na qual o excesso de cálcio desloca o potássio do complexo de troca, permitindo que o mesmo seja passível de ser lixiviado (Medeiros et al., 2008).

\section{Conclusão}

A irrigação com água subterrânea promove aumento dos teores de cálcio trocável no perfil do solo analisado (0-0,8 m), a elevação do $\mathrm{pH}$ e a redução da disponibilidade do manganês na camada superficial $(0-0,2 \mathrm{~m})$;

O solo irrigado com água subterrânea se apresenta com maior capacidade de troca de cátions;

A água subterrânea aumenta a relação $\mathrm{Ca} / \mathrm{Mg}$ e $\mathrm{Ca} / \mathrm{K}$, no solo cultivado com mangueira 'Palmer'.

\section{Referências}

Ayers, R. S; Westcot, D. W. A qualidade de água na agricultura. $2^{\mathrm{a}}$ ed. UFPB, Campina Grande, (FAO - Estudos Irrigação e Drenagem, 29). 1999. 153 p.

Allen, R. G.; Pereira, L. S.; Raes, D.; Smith, M. Evapotranspiración del cultivo: guías para la determinación de los requerimientos de agua de los cultivos. Rome: Food \& Agriculture Org., 2006. (Boletim FAO 56).
Almeida, E. V. de; Fernandes, F. M.; Boliani, A. C. Distribuição do sistema radicular do portaenxerto Coquinho sob copa da mangueira cv. Haden. Revista Brasileira de Fruticultura, v. 31, n. 3, p. 798-804, 2009. http://dx.doi.org/10.1590/S0100-29452009000300025

Anuário brasileiro da fruticultura 2018. Santa Cruz do Sul: Editora Gazeta Santa Cruz, 2018. 49p.

Apha [American Public Health Association]; Awwa [American Water Works Association]; Wef [Water Environment Federation]. Standard methods for the examination of water and wastewater. $22^{\mathrm{a}}$ ed. Washington: APHA/AWWA/WEF, 2012. $1360 \mathrm{p}$.

Associação central dos Fruticultores do Norte de Minas e Sudoeste Da bahia (ABANORTE). Levantamento e estudo da cultura da manga para viabilização deste ramo de agronegócio no norte de Minas Gerais. Janaúba, 2014.

Bernardo, S.; Soares, A. A.; Mantovani, E. C. Manual de Irrigação. $8^{a}$ ed. Viçosa: Editora UFV, 2006. 625p.

Brito, M. E. B.; Fernandes, P. D.; Gheyi, H. R.; Melo, A. S. de; Filho, W. S. S.; Santos, R. T. de. Sensibilidade à salinidade de híbridos trifoliados e outros porta-enxertos de citros. Revista Caatinga, v. 27, n. 1, p.17-27, 2014. https://periodicos.ufersa.edu.br/index.php/caatinga/article /view/2610

Carmona, F. de C.; Anghinoni, I.; Holzschuh, M. J.; Andrighetti, M. H. Cation dynamics in soils with different salinity levels growing irrigated rice. Revista Brasileira de Ciência do Solo, v. 34, n. 6, p. 1851-1863, 2010. http://dx.doi.org/10.1590/S010006832010000600009 
Carvalho, L. L. S.; Lacerda, C. F.; Andrade, E. M.; Lopes, F. B.; Junior, M. V.; Carvalho, C. M. Variabilidade espacial e temporal da qualidade da água de poços no perímetro irrigado do baixo Acaraú - CE. Revista Brasileira de Agricultura Irrigada v. 11, n. 2, p. 1348-1357, 2017.

https://inovagri.org.br/revista/index.php/rbai/article/view/ 623

Carvalho Filho, A.; Curi, N.; Marques, J. J. G. S. M. de; Shinzato, E.; Freitas D. A. F. de; Jesus, E. A. de; Massahud, R. T. L. R. Óxido de manganês em solos no quadrilátero ferrífero (MG). Revista Brasileira Ciências Solo, v. 35, n. 3, p. 793-804, 2011. http://dx.doi.org/10.1590/S0100-06832011000300015

Faria, F. H. de S.; Lima, L. A.; Ribeiro, M. S.; Santos, S. R.; Ribeiro, K. M. Avaliação da salinidade, sodicidade e alcalinidade das águas subterrâneas para irrigação em Jaíba e Janaúba, Minas Gerais. Irriga, v. 14, n. 3, p. 299-313, 2009. https://doi.org/10.15809/irriga.2009v14n3p299313

Fornes, F.; Garcia-de-la-Fuente, R.; Belda, R.M.;Abad, M. "Alperjo" compost amendment of contaminated calcareous and acidic soils: effects on growth and trace element uptake by Five Brassica species. Bioresource Technology, v. 100, n. 17, p. 3982-3990, 2009. https://doi.org/10.1016/j.biortech.2009.03.050

Holanda, J. S.; Amorim, J. R. A.; Neto, M. F.; Holanda, A. C. Qualidade da água para irrigação. In: Gheyi, H. R.; Dias, N. S.; Lacerda, C. F. (ed.) Manejo da salinidade na agricultura: Estudos básicos e aplicados. 2 ${ }^{\mathrm{a}}$ ed, Fortaleza: INCT Sal, 2016. Cap. 4, p. 35-50.

Hue, N. V.; Vega, S.; Silva, J. M. Manganese toxicity in a Hawaiian Oxisol affected by soil $\mathrm{pH}$ and organic amendments. Soil Science Society of America Journal Abstract., v. 65, n. $1, \quad$ p. $153-160, \quad 2001$. http://dx.doi.org/10.2136/sssaj2001.651153x

Iglesias, M.; Uhlein, A. Estratigrafia do Grupo Bambuí e coberturas fanerozóicas no vale do rio São Francisco, Norte de Minas Gerais. Revista Brasileira de Geociências, v. 39, n. 2, p. 256266, 2009. http://ppegeo.igc.usp.br/index.php/rbg/article/view/7661

Lepsch, I. F. 19 lições de pedologia. São Paulo: Oficina de textos, 2011. 455p.

Lucena, C. C. de; Siqueira, D. L. de; Martinez, H. E. P; Cecon, P. R. C. Salt stress change chlorophyll fluorescence in mango. Revista Brasileira de Fruticultura, v. 34, n. 4, p. 12451255, 2012. http://dx.doi.org/10.1590/S010029452012000400034

Malavolta, E.; Vitti, G. C.; Oliveira, S. A. Avaliação do estado nutricional das plantas: princípios e aplicações. $2^{\mathrm{a}}$ ed. Piracicaba: Associação Brasileira para Pesquisa da Potassa e do Fosfato, 1997. 319p.

Maluf, J. G. M.; Ghini, R.; Melo, L. B. B.; Silva, C. A. Fertilidade do solo e estado nutricional do cafeeiro cultivado em atmosfera enriquecida com $\mathrm{CO}_{2}$. Pesquisa Agropecuária Brasileira, v. 50, n. 11, p. 1087-1096, 2015. http://dx.doi.org/10.1590/S0100-204X2015001100012

Medeiros, J. F.; Neto, C. P. C. T.; Dias, N. S.; Gheyi, H. R.; Silva, M. V. T.; Loiola, A. T. Salinidade e $\mathrm{pH}$ de um Argissolo irrigado com água salina sob estratégias de manejo. Revista Brasileira de Agricultura Irrigada, v. 11, n. 3, p. 1407-1419, 2017. http://www.inovagri.org.br/revista/index.php/rbai/article/ view/560

Medeiros, J. C.; Albuquerque, J. A.; Mafra, A. L.; Rosa, J. D.; Gatiboni, L. C. Relação cálcio:magnésio do corretivo da acidez do solo na nutrição e no desenvolvimento inicial de plantas de milho em um Cambissolo Húmico Álico. Semina: Ciências Agrárias, v. 29, n. 4, p. 799-806, 2008. http://dx.doi.org/10.5433/16790359.2008v29n4p799

Nunes, R. L. C. Dias, N. S. da; Moura, C. F. de; Neto, O. N. S. de; Costa, J. M. da. Efeitos da salinidade da solução nutritiva na produção de pimentão cultivado em substrato de fibra de coco. Revista Caatinga, v. 26, n. 4, p. 48-53, 2013.

https://periodicos.ufersa.edu.br/index.php/caatinga/article /view/2734

Nunes, W. A. G. de A. Ker, J. C.; Neves, J. C. L.; Ruiz, H. A.; Beirigo, R. M.; Boncompani, A. L. P. Características químicas de solos da região de Janaúba, MG, irrigados com água de poços tubulares e do rio Gorutuba. Revista Brasileira de Ciência do Solo, v. 32, n. 1, p. 227-236, 2008. 06832008000100022 http://dx.doi.org/10.1590/S0100-

Nunes, W. A. G. de A. Ker, J. C.; Neves, J. C. L.; Ruiz, H. A.; Freitas, G. A. \& Beirigo, R. M. Qualidade da água de irrigação de poços tubulares e do rio gorutuba na região de 
Janaúba-MG. Irriga, v. 10, n. 4, p. 403-410, 2005. https://doi.org/10.15809/irriga.2005v10n4p419426

Pavinato, P. S.; Rosolem, C. A. Disponibilidade de nutrientes no solo: decomposição e liberação de compostos orgânicos de resíduos vegetais. Revista Brasileira de Ciência do Solo, v. 32, n. 3 , p. 911-920, 2008. http://dx.doi.org/10.1590/S0100-06832008000300001

Pegoraro, R. F.; Silva, I. R. ; Novais, R. F. ; Mendonça, E. S.; Gebrim, F. O.; Moreira, F. F. Fluxo difusivo e biodisponibilidade de zinco, cobre, ferro e manganês no solo: influência da calagem, textura do solo e resíduos vegetais. Revista Brasileira Ciência Solo, v. 30, n. 5, p. 859-868, 2006. http://dx.doi.org/10.1590/S010006832006000500012

Pinto, A. C. Q.; Costa, J. G.; Santos, C. A. F. Principais variedades. In: Genu, P. J. C.; Pinto, A. C. Q. (Eds). A cultura da mangueira. Embrapa Informação Tecnológica, Brasilia, 2002. Cap. 5, p. 94-116.

Pizarro, F. Riegos localizados de alta frecuencia (RLAF). Goteo, microaspersión,exudación. 3 ed. rev. y amp. Madrid: Ediciones MundiPrensa, 1996. 513p.

Raij, B.van; Cantarella, H.; Quaggio, J. A.; Furlani, A. M. C. Recomendações de adubação e calagem para o Estado de São Paulo. Campinas: IAC, 1996. 184p.
Porto Filho, Q.; Medeiros, J. F.; Gheyi, H. R.; Dias, N. S.; Sousa, P S.; Dantas, D. C.; Evolução da salinidade e pH de solo sob cultivo de melão irrigado com água salina. Revista Brasileira de Engenharia Agrícola e Ambiental, v. 15, n. 11, p. 1130-1137, 2011. http://dx.doi.org/10.1590/S1415-43662011001100004

Santos, M. de F. G. dos; Oliveira, F. A. de; Cavalcante, L. F.; Medeiros, J. F. de; Souza, C. C. de. Solo sódico tratado com gesso agrícola, composto de lixo urbano e vinhaça. Revista Brasileira de Engenharia Agrícola e Ambiental, v. 9, n. 3, p. 307-313, 2005. http://dx.doi.org/10.1590/S1415-43662005000300003

Santos, M. R. dos; Donato, S. L. R.; Coelho, E. F.; Cotrim Junior, P. R. F.; Castro, I. N. de. Irrigation deficit strategies on physiological and productive parameters of "tommy atkins" mango. Revista Caatinga, v. 29, n. 1, p. 173182, 2016. http://dx.doi.org/10.1590/198321252016v29n120rc

Teixeira, P. C.; Donagema, G. C.; Fontana, A.; Teixeira, W. G. T. Manual de Métodos de Análise de Solo. $3^{\mathrm{a}}$ ed. Brasília: Embrapa, 2017. $573 p$.

Valadares, R. V.; Valadares, S. V.; Fernandes, L. A.; Sampaio, R. A. Teores de nutrientes no solo e nutrição mineral do milho em áreas irrigadas com água calcária. Revista Caatinga, v. 27, n. 3, p. 169-176, 2014. https://periodicos.ufersa.edu.br/index.php/caatinga/article /view/2736 